\title{
Haploid embryonic stem cells: an ideal tool for mammalian genetic analyses
}

\author{
Linyu Shi, Hui Yang, Jinsong $\mathrm{Li}^{\bowtie}$ \\ Group of Epigenetic Reprogramming, State Key Laboratory of Cell Biology, Institute of Biochemistry and Cell Biology, Chinese \\ Academy of Sciences, Shanghai 200031, China \\ $\triangle$ Correspondence: jsli@sibs.ac.cn \\ Received September 3, 2010 Accepted September 12, 2012
}

\begin{abstract}
Identification of the function of all genes in the mammalian genome is critical in understanding basic mechanisms of biology. However, the diploidy of mammalian somatic cells has greatly hindered efforts to elucidate the gene function in numerous biological processes by mutagenesis-based genetic approaches. Recently, mouse haploid embryonic stem (haES) cells have been successfully isolated from parthenogenetic and androgenetic embryos, providing an ideal tool for genetic analyses. In these studies, mouse haES cells have already shown that they could be used in cell-based forward or reverse genetic screenings and in generating gene-targeting via homologous recombination. In particular, haES cells from androgenetic embryos can be employed as novel, renewable form of fertilization agent for yielding live-born mice via injection into oocytes, thus showing the possibility that genetic analysis can be extended from cellular level to organism level.
\end{abstract}

KEYWORDS haploid embryonic stem cells, parthenogenetic embryos, androgenetic embryos, genetic screening, diploid, genomic imprinting

\section{INTRODUCTION}

Most somatic cells in mammalian organisms are diploid, containing duplicate sets of chromosomes, a situation that is beneficial for their survival since if one copy of a gene is disrupted, the other copy can compensate for it. However, diploidy limits the identifying the function of a specific gene, because both alleles of the gene have to be mutated to generate a recessive homozygous. To resolve this problem, the haploid fungal spores of Saccharomyces cerevisiae have been largely utilized for mutational screens on a genome-wide scale, as a recessive mutation should result in an unambiguous phenotype due to the absence of a second gene copy.

In vertebrate, haploidy exists only in the postmeiotic germline, which is structurally specialized for fertilization. These cells cannot divide in vitro, thus preventing gene manipulation. Stable cultured haploid cell lines can be successfully derived from the embryos of some lower vertebrates, such as frogs (Freed and Mezger-Freed, 1970) and fish (Yi et al., 2009). Meanwhile, Zebrafish, as an important model organism for the study of early vertebrate development, its haploid embryos are amenable to insertional mutation and genetic screening (Wiellette et al., 2004). In mammalian organisms, the near-haploid human cell lines have been established from tumors (Kotecki et al., 1999; Sukov et al., 2010). Although these cells have been used for genetic screenings (Carette et al., 2009, 2011), their aneuploid karyotype and cancerous characteristics limits their application. Recently, three independent laboratories reported the isolation of mouse haES cells, with pilot genetic screenings and construction of a gene knockout (KO) mouse (Elling et al., 2011; Leeb and Wutz, 2011; Yang et al., 2012), representing an important breakthrough in the field.

Here, we review recent progress in derivation of mouse haES cells, discuss their potential applications and outline future challenges.

\section{DERIVATION OF haES CELL LINES}

There are two different kinds of haploid embryos according to their genetic origin, i.e., parthenogenetic and androgenetic embryos. To generate parthenogenetic haploid embryos, mature oocytes are activated by physical or chemical tech- 
niques (Paffoni et al., 2008), followed by the second polar body exclusion; the resulting embryos contain only one set of chromosome originated from oocyte. The other approach to make parthenogenetic haploid embryos is to remove the male pronucleus from the zygote. To generated androgenetic haploid embryos, nuclear transfer is employed to replace the chromosome-spindle-complex of a mature oocyte with a sperm head; the resulting embryos contain only one copy of paternal genome from sperm. The androgenetic haploid embryos can also be made by removal of the female pronucleus from the zygotes.

The first mammalian ES cells were derived from mouse embryos more than thirty years ago (Evans and Kaufman, 1981). Shortly after that, the same group attempted to derive haES cells from mouse haploid embryos (Kaufman et al., 1983). They generated 4 ES cell lines, however, all of them displayed a diploid karyotype (Kaufman et al., 1983). These results indicated that haploid cells convert to diploid spontaneously and haploidy might not be possible to be sustained in normal mammalian cells in vitro. Interestingly, haploid cells seem less compatible during differentiation and development, and haploid cell tends to become diploidy in vivo after transplantation of haploid embryos into pseudopregrant females (Ito et al., 1991; Kaufman, 1978; Latham et al., 2002).
In 2011, two high-profile papers reported the generation of mouse haES cells from parthenogenetic embryos (Elling et al., 2011; Leeb and Wutz, 2011). Several months later, haES cells were generated from androgenetic embryos (Yang et al., 2012). These successes were made possible by haploid cell enrichment using fluorescence-activated cell sorting (FACS) (Elling et al., 2011; Leeb and Wutz, 2011). HaES cells showed a similar colony morphology as normal mouse diploid ES cells, expressed classical ES cell markers, and contributed to various tissues (Elling et al., 2011; Leeb and Wutz, 2011) including the germline (Yang et al., 2012) upon injection into diploid blastocysts. Furthermore, these cells sustained genome integrity and maintained stable haploidy in vitro by repeated cell sorting.

\section{APPLICATIONS OF haES CELLS}

The motivation behind the generation of mammalian haES cells is to facilitate genetic studies. Recent reports (Elling et al., 2011; Leeb and Wutz, 2011; Yang et al., 2012) have shown preliminary applications of haES cells for genetic analyses without the limitation of normal diploid ES cells. Next we describe the applications of haES cells at both the cellular level and animal level (Fig. 1).
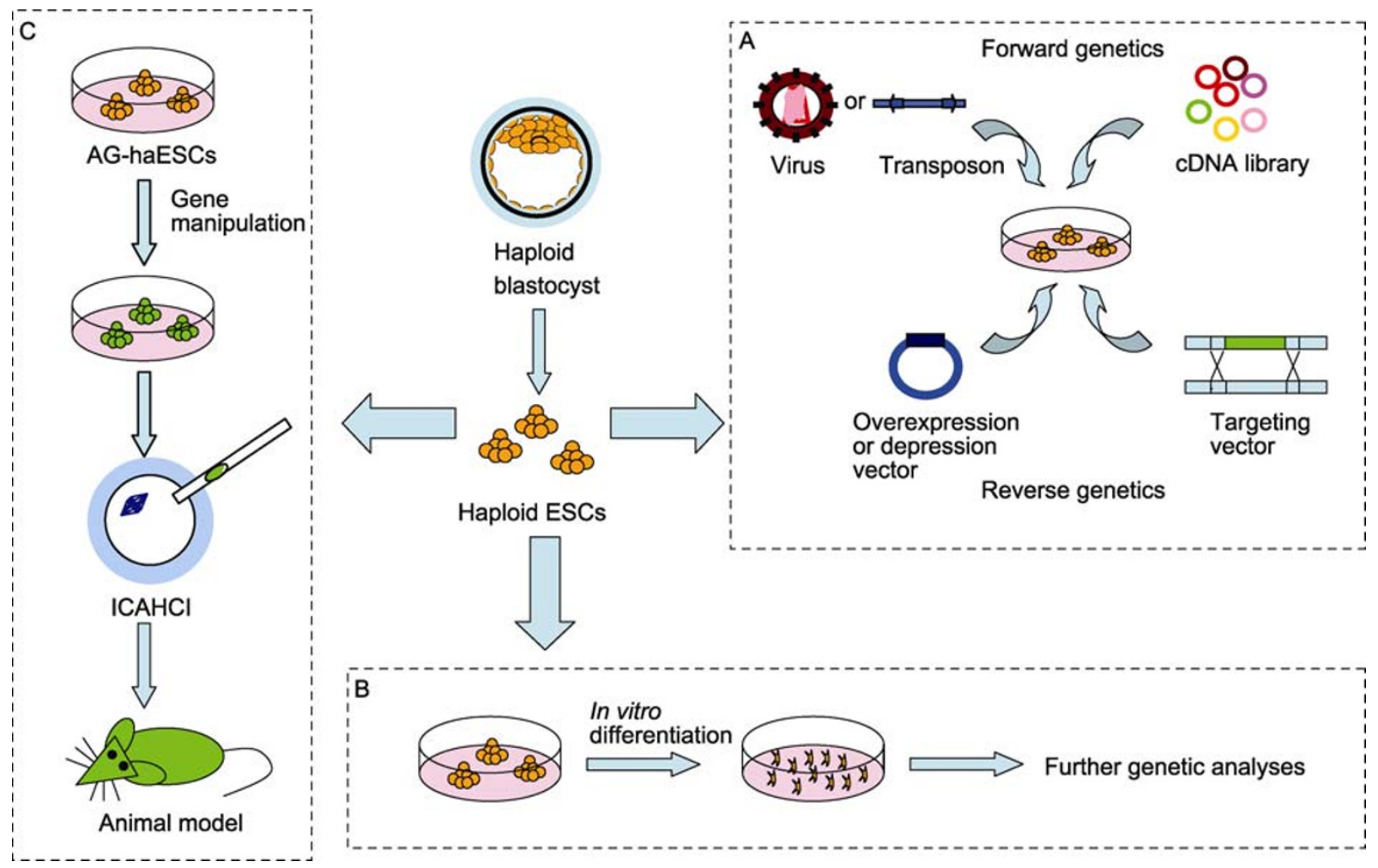

Figure 1. The applications of haES cells. Mammalian haES cells show their great potential for genetic analyses. (A) At the cellular level, the haES cells have proven to be widely applicable in forward and reverse genetics; $(B)$ the differentiated haploid cells could also be used for further genetic analyses. (C) At the organism level, AG-haES cells will be valuable tools in producing genetically modified animals for genome-wide screening or specific gene analysis. 


\section{At the cellular level}

The aim of forward and reverse genetic screenings is to discover the function of genes. Forward genetic screening is an approach used to identify some of the genes responsible for the phenotype of interest, and reverse screening is used to analyze the phenotype by disrupting a specific gene.

Transposon and retroviral insertional vectors were used to test the possibility of using haES cells for forward genetics (Elling et al., 2011; Leeb and Wutz, 2011). The authors adapted a similar loss-of-function screen strategy to select the relative drug-resistant ES cell clones. They identified some important genes after determining the insertion sites by PCR from ES cell clones selected. In addition, Elling et al. (2011) also described the application of mouse haES cells in reverse genetics. They designed an ingenious reversible gene trap vector for this purpose. The haES cells carrying the retroviral vector in the antisense orientation would not affect gene transcription, whereas transient Cre expression leading to convert the splice acceptor into the sense orientation would cause the truncation of the transcript. Thus, the phenotypic variation of the particular cell lines before and after Cre expression can be observed.

Furthermore, Yang et al. demonstrated that gene targeting via homologous recombination is feasible in haES cells, leading to successful generation of KO haES cells (Yang et al., 2012). Theoretically, KO haES cells could also be used for further gene screening by introduction of a cDNA library of expression plasmids or random insertional mutations, such as complementation screenings or synthetic lethal screenings (Grimm, 2004). Since mouse haES cells possess the ability to differentiate various cells of the three germ layers, an intriguing application of haES cells is to screen the key genes involved in a specific cell fate determination during differentiation (Schimenti, 2011).

\section{At the organism level}

An important outcome of genetic screening analyses is the establishment of animal model, thus the function of a specific gene could be evaluated at organism level in vivo. Forward and reverse genetic screenings have been successfully applied in parthenogenetic haES cells (Elling et al., 2011; Leeb and Wutz, 2011), however, the limitation of these haES cells is that successful conversion of the ES cells into live animals that would bear the ES cell-carried trait, has not been demonstrated (Elling et al., 2011; Leeb and Wutz, 2011). Yang et al. (2012) showed that AG-haES cells can be used as novel, renewable form of fertilization agent for yielding live-born mice (semicloned mice, SC mice) via injection into oocytes (intracytoplasmic AG-haES cell injection, ICAHCI), providing a new route to generate genetic-modified mouse model. They further obtained a F1 live-born pup after injection of the gene-targeted haES cells into oocytes and proved that this pup carried the targeted allele. Although this pup died shortly after birth, these results provide the proof of principle that genetically modified animals can be obtained via the ICAHCl technology using AG-haES cells that harbor a modified allele.

One caveat of AG-haES cells is that all SC mice generated via $\mathrm{ICAHCl}$ are females since the only the $X$ chromosome-bearing AG-haES cell lines can be generated, due to $Y$ chromosome-bearing androgenetic embryos could not develop to the blastocyst stage (Latham et al., 2002, 2000). Nevertheless, there are still several advantages when AG-haES cells and ICAHCI technology are used for generating genetic-modified mice. First, the biggest advantage of using AG-haES cells is the quick and reliable generation of F1 mice (SC mice). Therefore, it is not necessary to wait germline transmission from chimeras generated by traditional strategy using diploid ES cells, which is always the rate-limited step in both conventional and conditional gene knock-out experiments (Fig. 2). Second, for gene knock-in experiments, we can observe phenotype immediately in SC mice. In summary, the application of AG-haES cells will provide a new and simple method in the production of genetic modified mice.

An intriguing application of AG-haES cells is the generation of gene-modified animals by injection them into oocyts in other species where conventional ES cells can be obtained but fail to support the production of chimeras, therefore cannot obtain gene-modified animals, such as the monkey (Tachibana et al., 2012). Moreover, for large animals, it is hard to have many chimeric animals and do lots of mating to get germline transmission by chance. In contrast, once a single F1 SC animal from AG-haES cells is obtained, it is certain to get a transmission into offspring with limited breeding as half of the progeny will inherit the genetic modification.

Another potential advantage is that the application of AG-haES cells is similar to artificial fertilization technologies, such as intracytoplasmic sperm injection (ICSI), which is commonly used in human. For human reproduction, although it is not clear now whether human AG-haES cells could be generated and they would be as good as sperm for the purposes of artificially fertilization, the $\mathrm{ICAHCl}$ technique might ultimately be used in assisted human reproduction for those affected by genetic disease.

\section{CHALLENGES OF haES CELLS}

Mouse haES cells are amenable to efficient functional screening for genes involved in different biological processes; however, several technical hurdles need to be overcome in order to facilitate the potential applications of these cells.

It is noteworthy that the mouse haES cells convert to diploid spontaneously in vivo and in vitro (Elling et al., 2011; Leeb and Wutz, 2011; Yang et al., 2012). The haploidy maintenance is dependent on periodic purification by cell 


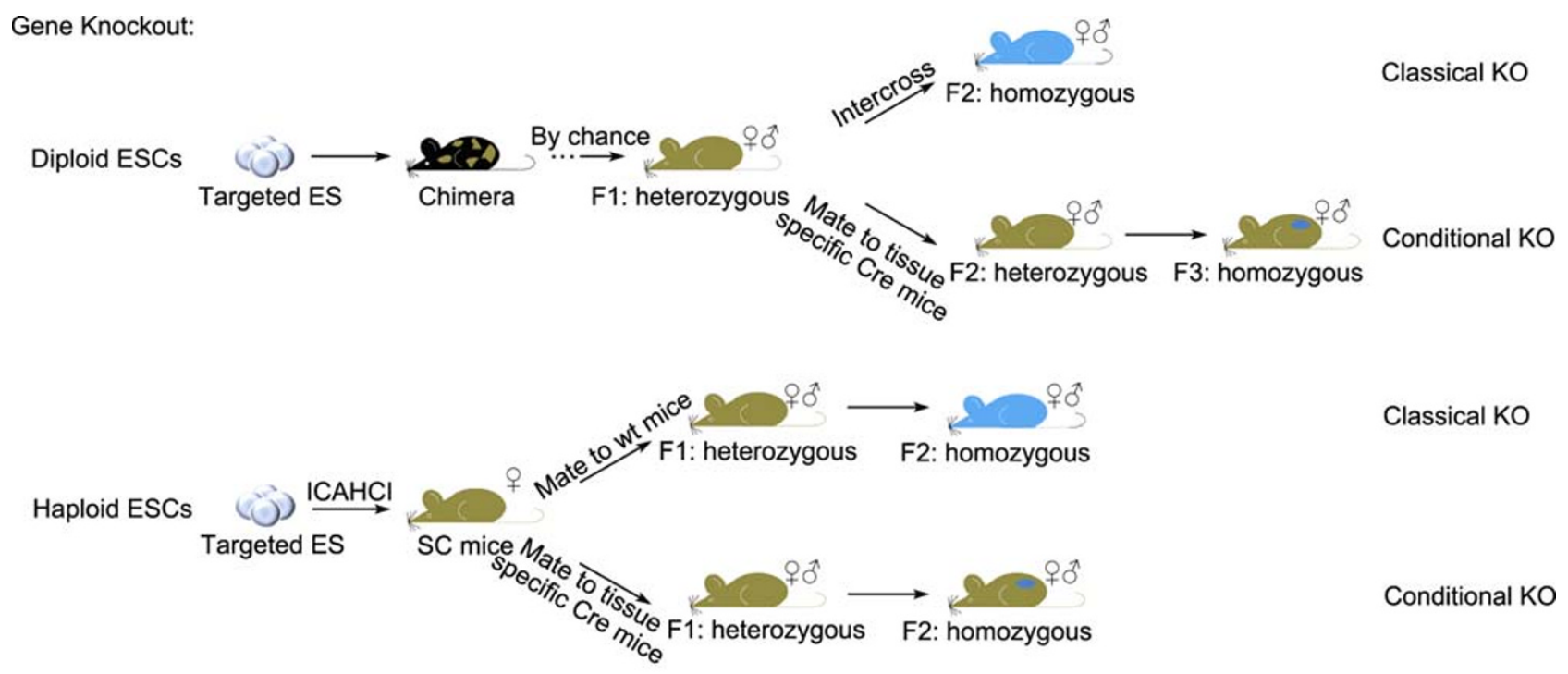

Gene Knock-in

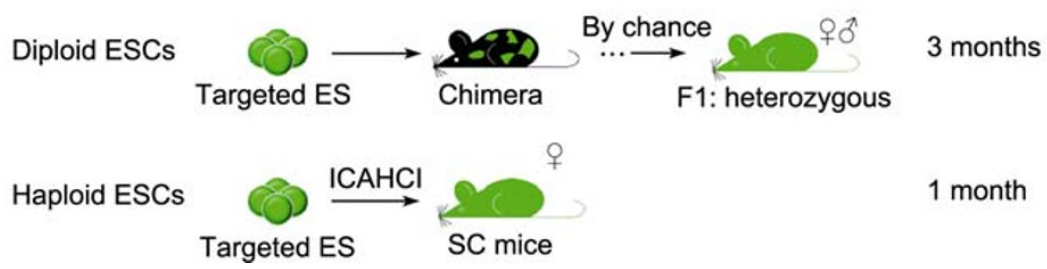

Figure 2. The applications of AG-haES cells in the production of Knockout and Knock-in mice (provided by Bang-an Wang). There are several advantages of AG-haES cells and ICAHCI in making genetic modified mice, including: 1) quick and reliable generation of F1 mice; 2) immediate phenotype in gene knock-in experiments.

sorting every several passages. For this purpose, the cells are stained by nuclear dye, Hoechst 33342, and the cells with a haploid DNA content are sorted and enriched by exposure to ultraviolet (UV) light. However, this repeated UV light exposure may cause intracellular damage (Erba et al., 1988) and cell apoptosis (Zhang et al., 1999; Zhang and Kiechle, 1998). Furthermore, nuclear staining by Hoechst may impair the developmental competence of embryos (Li et al., 2004; Maside et al., 2011; Versieren et al., 2012). The intriguing questions are why and how haES cells become into diploid spontaneously; which genes or signaling are involved in this conversion; and whether it is possible to maintain haploidy in vitro without using FACS.

Mouse haES cells are generated from parthenogenetic or androgenetic embryos, whose genetic materials are originated from maternal or paternal gametes, respectively. Meanwhile, since parental imprints established at the primordial germ cell stage are propagated through fertilization and persist during embryonic development, it is interesting to examine parental imprinting state in these cells. As expected, parental genomic imprints can be generally maintained in AG-haES cells (Yang et al., 2012), while imprinting state is not mentioned in haES cells from parthenogeneitc embryos (Elling et al., 2011; Leeb and Wutz, 2011). The maintenance of normal imprints in AG-haES cells will likely determine that AG-haES cells can contribute to the germline after injection into diploid blastocyst (Yang et al., 2012) and can support the full-term development of embryos produced by injection into oocytes (Yang et al., 2012). Nevertheless, H19, a paternal imprinted gene, which should not be expressed in sperm, thus not expressed in AG-haES cell, is upregulated in AG-haES cells (Yang et al., 2012). The loss of $H 19$ imprint in hAG-haES cells might account for the low birth rate of SC mouse and probably for growth-retarded fetuses by the following observations: 1) methylation variation at the $\mathrm{H} 19$ imprinted gene is seen in AG-haES cells and the smaller $\mathrm{SC}$ pups born from ICAHCl are characterized by hypomethylation at $H 19$; 2) growth-retarded pups were born at a higher frequency from ICAHCl using the cells of later passages that had severer loss of the $\mathrm{H} 19$ methylation imprint; 3) the loss of $\mathrm{H} 19$ imprinting was observed in both $\mathrm{KO}$ AG-haES cells and the resulting retarded SC pup born after ICAHCl. Thus, a future task is to define the appropriate derivation and culture conditions that would enable the relatively stable maintenance of imprinting state in haploid ES cells. 


\section{CONCLUDING REMARKS}

The generation of haES cells from mouse parthenogenetic and androgenetic embryos provides an ideal tool for genetic analyses. Remarkably, the sperm-like characteristics of AG-haES cells enable them artificially "fertilize" oocytes, making it easier to produce genetically modified mice. It is worthwhile to investigate how to stabilize the haploidy and imprinting state in mouse haES cells and whether haES cells could be derived from other species in future.

\section{ACKNOWLEDGEMENTS}

This study was supported by grants from the Ministry of Science and Technology of China (No. 2009CB941101 to J.L.), and the "Strategic Priority Research Program" of the Chinese Academy of Sciences (No. XDA01010403 to J.L.).

\section{REFERENCES}

Carette, J.E., Guimaraes, C.P., Varadarajan, M., Park, A.S., Wuethrich, I., Godarova, A., Kotecki, M., Cochran, B.H., Spooner, E., Ploegh, H.L., et al. (2009). Haploid genetic screens in human cells identify host factors used by pathogens. Science 326 , 1231-1235.

Carette, J.E., Guimaraes, C.P., Wuethrich, I., Blomen, V.A., Varadarajan, M., Sun, C., Bell, G., Yuan, B., Muellner, M.K., Nijman, S.M., et al. (2011). Global gene disruption in human cells to assign genes to phenotypes by deep sequencing. Nat Biotech 29, 542-546.

Elling, U., Taubenschmid, J., Wirnsberger, G., O'Malley, R., Demers, S.P., Vanhaelen, Q., Shukalyuk, A.I., Schmauss, G., Schramek, D., Schnuetgen, F., et al. (2011). Forward and reverse genetics through derivation of haploid mouse embryonic stem cells. Cell Stem Cell 9, 563-574.

Erba, E., Ubezio, P., Broggini, M., Ponti, M., and D'Incalci, M. (1988). DNA damage, cytotoxic effect and cell-cycle perturbation of Hoechst 33342 on L1210 cells in vitro. Cytometry 9, 1-6.

Evans, M.J., and Kaufman, M.H. (1981). Establishment in culture of pluripotential cells from mouse embryos. Nature 292, 154-156.

Freed, J.J., and Mezger-Freed, L. (1970). Stable haploid cultured cell lines from frog embryos. Proc Natl Acad Sci U S A 65, 337-344.

Grimm, S. (2004). The art and design of genetic screens: mammalian culture cells. Nat Rev Genet 5, 179-189.

Ito, M., Kaneko-Ishino, T., Ishino, F., Matsuhashi, M., Yokoyama, M., and Katsuki, M. (1991). Fate of haploid parthenogenetic cells in mouse chimeras during development. J Exp Zool 257, 178-183.

Kaufman, M.H. (1978). Chromosome analysis of early postimplantation presumptive haploid parthenogenetic mouse embryos. J Embryol Exp Morphol 45, 85-91.

Kaufman, M.H., Robertson, E.J., Handyside, A.H., and Evans, M.J. (1983). Establishment of pluripotential cell lines from haploid mouse embryos. J Embryol Exp Morphol 73, 249-261.

Kotecki, M., Reddy, P.S., and Cochran, B.H. (1999). Isolation and characterization of a near-haploid human cell line. Exp Cell Res 252, 273-280.

Latham, K.E., Akutsu, H., Patel, B., and Yanagimachi, R. (2002). Comparison of gene expression during preimplantation development between diploid and haploid mouse embryos. Biol Reprod 67, 386-392.

Latham, K.E., Patel, B., Bautista, F.D., and Hawes, S.M. (2000). Effects of $\mathrm{X}$ chromosome number and parental origin on X-linked gene expression in preimplantation mouse embryos. Biol Reprod $63,64-73$.

Leeb, M., and Wutz, A. (2011). Derivation of haploid embryonic stem cells from mouse embryos. Nature 479, 131-134.

Li, G.P., White, K.L., and Bunch, T.D. (2004). Review of enucleation methods and procedures used in animal cloning: state of the art. Cloning Stem Cells 6, 5-13.

Maside, C., Gil, M.A., Cuello, C., Sanchez-Osorio, J., Parrilla, I., Lucas, X., Caamano, J.N., Vazquez, J.M., Roca, J., and Martinez, E.A. (2011). Effects of Hoechst 33342 staining and ultraviolet irradiation on the developmental competence of in vitro-matured porcine oocytes. Theriogenology 76, 1667-1675.

Paffoni, A., Brevini, T.A., Gandolfi, F., and Ragni, G. (2008). Parthenogenetic activation: biology and applications in the ART laboratory. Placenta 29 Suppl B, 121-125.

Schimenti, J. (2011). Haploid embryonic stem cells and the dominance of recessive traits. Cell Stem Cell 9, 488-489.

Sukov, W.R., Ketterling, R.P., Wei, S., Monaghan, K., Blunden, P., Mazzara, P., Raghavan, R., Oliviera, A.M., Wiktor, A.E., Keeney, G.L., et al. (2010). Nearly identical near-haploid karyotype in a peritoneal mesothelioma and a retroperitoneal malignant peripheral nerve sheath tumor. Cancer Genet Cytogenet 202, 123-128.

Tachibana, M., Sparman, M., Ramsey, C., Ma, H., Lee, H.S., Penedo, M.C., and Mitalipov, S. (2012). Generation of chimeric rhesus monkeys. Cell 148, 285-295.

Versieren, K., Heindryckx, B., Qian, C., Gerris, J., and De Sutter, P. (2012). Toxic effects of Hoechst staining and UV irradiation on preimplantation development of parthenogenetically activated mouse oocytes. Zygote, 1-9.

Wiellette, E., Grinblat, Y., Austen, M., Hirsinger, E., Amsterdam, A., Walker, C., Westerfield, M., and Sive, H. (2004). Combined haploid and insertional mutation screen in the zebrafish. Genesis 40, 231-240.

Yang, H., Shi, L., Wang, B.A., Liang, D., Zhong, C., Liu, W., Nie, Y., Liu, J., Zhao, J., Gao, X., et al. (2012). Generation of genetically modified mice by oocyte injection of androgenetic haploid embryonic stem cells. Cell 149, 605-617.

Yi, M., Hong, N., and Hong, Y. (2009). Generation of medaka fish haploid embryonic stem cells. Science 326, 430-433.

Zhang, X., Chen, J., Davis, B., and Kiechle, F. (1999). Hoechst 33342 induces apoptosis in HL-60 cells and inhibits topoisomerase I in vivo. Arch Pathol Lab Med 123, 921-927.

Zhang, X., and Kiechle, F.L. (1998). Hoechst 33342 induces apoptosis and alters tata box binding protein/DNA complexes in nuclei from $\mathrm{BC} 3 \mathrm{H}-1$ myocytes. Biochem Biophys Res Commun $248,18-21$. 